“ (C) 2013 IEEE. Personal use of this material is permitted. Permission from IEEE must be obtained for all other uses, in any current or future media, including

reprinting/republishing this material for advertising or promotional purposes, creating new collective works, for resale or redistribution to servers or lists, or reuse of any copyrighted component of this work in other works." 


\title{
The Application of Discrete Sliding Mode Control in Parabolic PDE Dynamics
}

\author{
Ahmadreza Argha, Li Li, Steven W. Su and Hung Nguyen
}

\begin{abstract}
In this paper, the problem of applying Discrete Sliding Mode Control (DSMC) on spatially finite-dimensional systems arising from discretization of bi-variate Partial Differential Equations (PDEs) describing spatio-temporal systems is studied. To this end, heat transfer PDE is discretized to create 2D discrete dynamics and eventually this 2D spatiotemporal discrete form is represented in $1 \mathrm{D}$ vectorial form. In order to study the effect of discrepancy between original PDE dynamics and their discrete schemes, an uncertainty term is also considered for the obtained discrete dynamics. According to the notion of strong stability and, in addition, using scaling matrices (similarity transformation), a new method for considering the stability of discrete-time systems in the presence of general uncertainty term (matched and unmatched) is developed. It is also shown that the proposed method in this paper can be used for the case with spatial constraints on the actuation. Consequently, as special cases, the problem of spatially piecewise constant, sparse and also boundary control input are studied.
\end{abstract}

\section{INTRODUCTION}

Although PDEs problem are usually much harder to address than ODEs, discretizing method which converts PDEs to linear 2D systems can be used as an alternative simple solution [1]. In this paper, we will use the discrete finite-dimensional approximation (numerical approximation) of infinite-dimensional parabolic PDEs to deploy the Discrete Sliding Mode Control (DSMC) method to the dynamics defined by PDEs.

There are a number of studies on the dynamics described by PDEs. For instance, in [2] - [3], controlling an unstable linear system with a wave PDE in the actuation path is studied. Moreover, Iterative Learning Control (ILC) is used in [4] for a distributed parameter system which is governed by a second order hyperbolic PDE. In [5], the ILC method is deployed to distributed parameter systems governed by parabolic PDEs. In [6], an irrigation application in dry-land farming governed by a set of PDEs which include three spatial variables and one temporal independent variable is explained. In [7], ILC method is applied to parabolic classes of spatio-temporal systems based on the stability theory of 2D discrete systems. Basically, Sliding Mode Control (SMC) is a nonlinear control method and a special kind of Variable Structure Control (VSC) which can change its structure automatically in order to improve the dynamic performance. The main idea of SMC is to force the states into the sliding surface and remain

Ahmadreza Argha, Li Li, Steven W. Su and Hung Nguyen are with Faculty of Engineering and Information Technology, University of Technology, Sydney, PO Box 123, Broadway, NSW 2007, Australia.

\{Ahmadreza.Argha, Li.Li, Steven.Su, Hung. Nguyen $\}$ euts. edu.au on the sliding surface after reaching. The most significant characteristics of SMC is its robustness, as it provides dynamics with an invariance property to matched uncertainties [8]. Therefore, SMC can address the modeling uncertainties and especially it is useful when the exact model of the dynamics is not available. However, there is a significant drawback for Continuous SMC (which mainly involves a discontinuous control term) which is known as chattering. Mainly, the method of softening the discontinuous control part by a continuous approximation is used for reducing the chattering [26]. On the other hand, for DSMC, elimination of the discontinuous control part from the control law leads to the eliminating the chattering issue [17], [20], [18]. The obtained control law is called linear control law.

Obviously, one approach to applying SMC to PDEs is to work directly with the determining equations; for instance, continuous SMC is used as a boundary control method for stabilizing infinite-dimensional parabolic PDE systems [9], and also with application to Arc Welding [10]. As an alternative method, one can discretize PDEs by an implicit discretization scheme which is appropriate for dynamics over spatially finite problems. In this paper, using DSMC a new method for dealing with the problem of stabilizing parabolic PDE dynamics via discretizing the heat PDEs by an implicit discretization scheme is proposed. To this end, after discretizing the PDE equation and making it a 2D system, it is converted to a 1D form with vector states, and then the $1 \mathrm{D}$ sliding mode control is used to stabilize the obtained dynamics.

On the other hand, physical constraints in many distributed parameter systems are arising from the fact that only boundary control can be used for controlling this kind of dynamics. However, distributed sensors and also actuators have been recently developed in a number of fields. Hence, distributed controllers have had effective application in infinitedimensional dynamics [7]. This can also justify our innovation to use DSMC for stabilizing the dynamics arised from heat PDEs which covers general actuation constraints. The problem is then extended to spatially constrained actuators, for more generality.

Besides, the discrepancy between the original PDE model and its discrete approximation which mainly depends on the space and time step sizes, makes it inevitable to consider an uncertainty term in the obtained discrete state space. With a glimpse into the literature, it can be found that DSMC is mainly used for the systems which contain matched uncertainty and/or disturbance only [17], [18]. In this paper, a more general case of uncertainty (matched and unmatched) 
including model-based uncertainty and external disturbance is considered. In order to achieve this goal, primarily, the notion of strong stability [23] is introduced. Then, it will be shown that any asymptotic stable system is strongly stable under a specific norm. Eventually, as a significant contribution, a new method to consider the bound on system states subject to the general uncertainty is given based on the notion of strong stability.

The rest of this paper is organized as follows: in Section II, the procedure of discretizing a heat PDE is explained. Section III describes the Discrete Sliding Mode Controller design for our problem. In Section IV, the notion of strong stability is introduced. Section V explains the DSMC for the obtained 1D discrete dynamics including general uncertainty term. In Section VI, effectiveness and efficiency of the proposed method is studied by numerical examples. Finally, Section VII concludes this paper.

\section{DISCRETIZING HEAT PDES}

This section intends to explain the method of discretizing the heat transfer equation (as a parabolic PDE). Consider the following heat transfer equation,

$$
\frac{\partial h(t, x)}{\partial t}=\Upsilon \frac{\partial^{2} h(t, x)}{\partial x^{2}}+\zeta u(t, x) \quad t \geq 0, \quad 0<x<l,
$$

where $h(t, x)$ and $u(t, x)$ represent the heat flow and the control input, respectively. Here, $t$ and $x$ are time and space variables respectively; thermal diffusivity $\Upsilon$ is a positive constant; $\zeta$ is an operator characterizing the actuation location constraints, which is independent of $t$. It is assumed that time dependent Dirichlet boundary conditions are imposed to the heat PDE (1),

$$
h(t, 0)=\hat{\alpha}(t), \quad h(t, l)=\hat{\beta}(t), \quad t \geq 0 .
$$

Besides, the following initial conditions are assumed,

$$
h(0, x)=g(x), \quad 0 \leq x \leq l .
$$

Whenever the direct discretization method is applied to heat transfer dynamics, it is necessary to guarantee the numerical stability by choosing proper sampling period. To discretize the equation (1), the following uniformly mesh is assumed,

$$
t_{k}=k \Delta t, x_{j}=j \Delta x
$$

where $\Delta t$ and $\Delta x=\frac{l}{n}$ represent, respectively, the time step size and the spatial mesh size and also $n$ represents the number of spatial meshes. From Taylor's expansion we can obtain [13]

$$
\begin{array}{r}
h(t \pm \Delta t, x)=h(t, x) \pm \Delta t \frac{\partial h(t, x)}{\partial t}+ \\
\frac{1}{2}(\Delta t)^{2} \frac{\partial^{2} h(t, x)}{\partial t^{2}}+O\left[(\Delta t)^{3}\right]
\end{array}
$$

Therefore

$$
\frac{\partial^{2} h\left(t_{k}, x_{j}\right)}{\partial x^{2}} \approx \frac{h\left(t_{k}, x_{j+1}\right)-2 h\left(t_{k}, x_{j}\right)+h\left(t_{k}, x_{j-1}\right)}{(\Delta x)^{2}}
$$

and similarly

$$
\frac{\partial h(t, x)}{\partial t} \approx \frac{h\left(t_{k+1}, x_{j}\right)-h\left(t_{k}, x_{j}\right)}{(\Delta t)} .
$$

Define the vectorial form $H(k)=\left[h_{k, 1}, h_{k, 2}, \ldots, h_{k, n-1}\right]^{T}=$ $\left[h\left(t_{k}, x_{1}\right), h\left(t_{k}, x_{2}\right), \ldots, h\left(t_{k}, x_{n-1}\right)\right]^{T}, \quad$ and $U(k)=$ $\left[u_{k, i_{1}}, u_{k, i_{2}}, \ldots, u_{k, i_{m}}\right]^{T}$, where $u_{k, i_{w}}, w=1, \cdots, m$ are control input at locations $x_{i_{w}}, w=1, \cdots, m$, and time $k$ which is specified by the operator $\zeta$ in (1). Then, we have

$$
H(k+1)=\Lambda H(k)+B U(k)+v(k),
$$

where

$\Lambda=\left[\begin{array}{ccccc}(1-2 \kappa) & \kappa & & & \\ \kappa & (1-2 \kappa) & \kappa & & \\ & \kappa & \ddots & \ddots & \\ & & \ddots & & \kappa \\ & & & \kappa & (1-2 \kappa)\end{array}\right], v(k)=\left[\begin{array}{c}\kappa \hat{\alpha}_{k} \\ 0 \\ \vdots \\ 0 \\ \kappa \hat{\beta}_{k}\end{array}\right]$,

$\kappa=\frac{\Upsilon \Delta t}{(\Delta x)^{2}}$ and $B \in R^{(n-1) \times m}$.

Remark 1: It is shown in [13] that this discretizing method is stable if $0 \leq \kappa \leq \frac{1}{2}$. Besides, an unconditionally stable method can be obtained by the Crank-Nicolson discretizing method [7].

In (7), the control matrix $B$ and control input $U(k)$ can be different based on different spatial constraints. According to the physical position of spatially distributed controller, it is assumed that there are only $m$ locations available for control. Thus, the significant point is that the number of spatially distributed states and control inputs are not necessarily equal. It should be noted that in [14], some examples of actuators (sensors) such as spatially distributed (zone case and pointwise case) and boundary (zone case and point-wise case) actuators for diffusion systems are considered. This will lead to more control options including spatially piece-wise constant, sparse and also boundary control etc.

For instance, in some applications, the control input is in spatially piece-wise constant form. As an illustration, assume that the control input has the same value at every successive two spatial points. In this case, the control vector is supposed to be $U(k)=\left[u_{k, 1}, u_{k, 3}, u_{k, 5}, \ldots\right]^{T}$ and also the control matrix is assumed to be as $B_{1}$ in (9). It is also possible that control effort is only possible in restricted points of space mesh named spatially sparse control input. As an illustration, for the control vector $U(k)=\left[u_{k, 1}, u_{k, 3}, u_{k, 5}, \ldots\right]^{T}$, the control matrix is as $B_{2}$ in the following,

$$
B_{1}=\left[\begin{array}{llll}
\mu & 0 & 0 & \\
\mu & 0 & 0 & \\
0 & \mu & 0 & \\
0 & \mu & 0 & \cdots \\
0 & 0 & \mu & \\
0 & 0 & \mu & \\
& & \vdots &
\end{array}\right], B_{2}=\left[\begin{array}{cccc}
\mu & 0 & 0 & \\
0 & 0 & 0 & \\
0 & \mu & 0 & \\
0 & 0 & 0 & \cdots \\
0 & 0 & \mu & \\
0 & 0 & 0 & \\
& & \vdots
\end{array}\right]
$$

Furthermore, as it was mentioned before, in many distributed parameter systems, owing to the physical constraints, 
boundary controllers are the only choice. In this case, we assume that in (7), boundary condition on one side is the control inputs of the system and as a result it is not constant value. To this end, system (7) is changed to the following form

$$
H(k+1)=\Lambda H(k)+B u_{b}(k)+\hat{v}(k),
$$

where

$$
\hat{v}(k)=\left[\begin{array}{llll}
\kappa \hat{\alpha}_{k} & 0 & \ldots & 0
\end{array}\right]^{T}, B=\left[\begin{array}{llll}
0 & 0 & \ldots & \mu
\end{array}\right]^{T},
$$

and $\hat{v} \in R^{n-1}, B \in R^{(n-1) \times 1}$. Here, $u_{b}(k)$ is a scalar which represents the control input on the boundary. Generally, based on the physical position of actuators, control matrix $B$ can have any different form, including the mentioned forms. In all these cases, it is assumed that matrix $B$ has full rank, $\operatorname{rank}(B)=m$.

\section{DESIGNING DSMC}

Discrete Sliding Mode Control (DSMC) was first introduced in the mid 80s [15]. This idea was followed by a large number of publications [16] - [19]. Similar to Continuous Sliding Mode Control (CSMC), the design procedure of the DSMC for stabilizing problems is split into two steps:

1) Design a sliding surface which should have stable internal dynamics.

2) Create a control law which drives the closed-loop system into the sliding surface and forces the system trajectories to stay on or at least as close as possible to the surface.

Here, the objective is to design a DSMC to stabilize the states of the dynamics in (7). Generally, it is assumed that $B \in R^{(n-1) \times m}$ and $m \leq n-1$. Obviously, the dynamics in (7) is not in regular form with control matrices (9). Besides, $\operatorname{rank}(B)=m$ (matrix $B$ has full rank) and it is assumed that the pair $(\Lambda, B)$ is controllable [19]. Since $\operatorname{rank}(B)=m$, there exists an orthogonal matrix $T_{r} \in R^{(n-1) \times(n-1)}$ such that

$$
T_{r} B=\left[\begin{array}{c}
0_{[(n-1)-m] \times m} \\
\bar{B}_{2}
\end{array}\right],
$$

where the matrix $\bar{B}_{2} \in R^{m \times m}$ is nonsingular [20] (The orthogonal matrix $T_{r}$ can be computed using $Q R$ decomposition [20]). After the coordinate transformation, the dynamics in (7) is converted to

$$
\begin{gathered}
{\left[\begin{array}{c}
\bar{h}_{1}(k+1) \\
\bar{h}_{2}(k+1)
\end{array}\right]=\left[\begin{array}{ll}
\bar{\Lambda}_{11} & \bar{\Lambda}_{12} \\
\bar{\Lambda}_{21} & \bar{\Lambda}_{22}
\end{array}\right]\left[\begin{array}{l}
\bar{h}_{1}(k) \\
\bar{h}_{2}(k)
\end{array}\right]+} \\
{\left[\begin{array}{c}
0_{[(n-1)-m] \times m} \\
\bar{B}_{2}
\end{array}\right] U(k)+T_{r} v(k),}
\end{gathered}
$$

where

$$
\begin{aligned}
& \bar{H}(k)=\left[\begin{array}{l}
\left.\bar{h}_{1}(k)\right] \\
\bar{h}_{2}(k)
\end{array}\right]=T_{r} H(k), \\
& \bar{h}_{1}(k) \in R^{[(n-1)-m]} \text { and } \bar{h}_{2}(k) \in R^{m} \\
& \bar{\Lambda}_{11} \in R^{[(n-1)-m] \times[(n-1)-m]}, \bar{\Lambda}_{12} \in R^{[(n-1)-m] \times m} \\
& \bar{\Lambda}_{21} \in R^{m \times[(n-1)-m]}, \bar{\Lambda}_{22} \in R^{m \times m} .
\end{aligned}
$$

To design the sliding surface, since the boundary conditions do not influence the stability situation, we can temporarily ignore the term $T_{r} v(k)$ arising from boundary conditions. Now, sliding surface is introduced as

$$
\sigma_{h}(k)=\bar{S} \bar{H}(k)=\bar{S}_{1} \bar{h}_{1}(k)+\bar{S}_{2} \bar{h}_{2}(k)
$$

where $\bar{S}_{1} \in R^{m \times[(n-1)-m]}$ and $\bar{S}_{2} \in R^{m \times m}$ are the design parameters which determine the sliding surface and should be chosen such that, in the case that $\sigma_{h}(k)=0$, all remaining dynamics are stable. During ideal sliding on the surface, $\sigma_{h}(k)=0$ for all $k \geq k_{s}$, where $k_{s}$ is the time when sliding starts, therefore

$$
\bar{h}_{2}(k)=-\bar{S}_{2}^{-1} \bar{S}_{1} \bar{h}_{1}(k)
$$

Substituting the equation (16) into the equation (13) leads to

$$
\bar{h}_{1}(k+1)=\left(\bar{\Lambda}_{11}-\bar{\Lambda}_{12} \bar{S}_{2}^{-1} \bar{S}_{1}\right) \bar{h}_{1}(k)
$$

Hence, stability in the sliding mode is satisfied when all eigenvalues of the matrix $\left(\bar{\Lambda}_{11}-\bar{\Lambda}_{12} \bar{S}_{2}^{-1} \bar{S}_{1}\right)$ are located inside the unit circle. On the other hand, in [26], it is presented that if the pair $(\Lambda, B)$ is controllable, the pair $\left(\bar{\Lambda}_{11}, \bar{\Lambda}_{12}\right)$ is controllable as well. Therefore, the problem of finding the term $\bar{S}_{2}^{-1} \bar{S}_{1}$ can be addressed as a classical state feedback problem which can be solved by pole placement, LQRdesign or LMI method. It is also assumed that the matrix $\bar{S}_{2}$ plays the role of a scaling parameter which can be selected arbitrarily but invertible. Here, we design $\bar{S}_{1}, \bar{S}_{2}$ by solving a certain discrete Riccati equation with proper choices of weighting matrices. Eventually, the sliding surface in the original coordinate can be found by

$$
S=\left[\begin{array}{ll}
\bar{S}_{1} & \bar{S}_{2}
\end{array}\right] T_{r}
$$

In this stage, we want to design a controller which ensures the sliding mode of system. Firstly, a transformation matrix $T_{S}$ is defined as

$$
T_{s}=\left[\begin{array}{cc}
I_{(n-1)-m} & 0_{[(n-1)-m] \times m} \\
\bar{S}_{1} & \bar{S}_{2}
\end{array}\right] .
$$

Using the above transformation matrix the dynamics in (13) is converted to

$$
\begin{array}{r}
\tilde{H}(k+1)=\left[\begin{array}{c}
\bar{h}_{1}(k+1) \\
\sigma_{h}(k+1)
\end{array}\right]=\left[\begin{array}{cc}
\tilde{\Lambda}_{11} & \tilde{\Lambda}_{12} \\
\tilde{\Lambda}_{21} & \tilde{\Lambda}_{22}
\end{array}\right]\left[\begin{array}{c}
\bar{h}_{1}(k) \\
\sigma_{h}(k)
\end{array}\right]+ \\
{\left[\begin{array}{c}
0_{[(n-1)-m] \times m} \\
\bar{S}_{2} \bar{B}_{2}
\end{array}\right] U(k)+\left[\begin{array}{c}
\tilde{v}_{1}(k) \\
\tilde{v}_{2}(k)
\end{array}\right],}
\end{array}
$$

where $\left[\begin{array}{ll}\tilde{v}_{1}^{T}(k) & \tilde{v}_{2}^{T}(k)\end{array}\right]^{T}=T_{s} T_{r} v(k)$ and $\tilde{v}_{1}(k) \in R^{(n-1)-m}$, $\tilde{v}_{2}(k) \in R^{m}$. In order to design a controller which forces the closed-loop system into the sliding mode we use the linear reaching law presented in [21] and [18], which is given by

$$
\sigma_{h}(k+1)=\Phi \sigma_{h}(k)
$$

where $\Phi \in R^{m \times m}$ has been chosen to be a diagonal matrix with all its diagonal elements $\phi_{r}, r=1, \ldots, m$, satisfying $0 \leq \phi_{r}<1$ 
Theorem 1: The system (20) is stable underc the control input $U$ as

$$
U(k)=\left(\bar{S}_{2} \bar{B}_{2}\right)^{-1}\left(\left[\Phi-\tilde{\Lambda}_{22}\right] \sigma_{h}(k)-\tilde{\Lambda}_{21} \bar{h}_{1}(k)-\tilde{v}_{2}(k)\right) .
$$

Proof: Applying the above control law to the system (20) results in the following closed-loop system

$$
\left[\begin{array}{c}
\bar{h}_{1}(k+1) \\
\sigma_{h}(k+1)
\end{array}\right]=\left[\begin{array}{cc}
\tilde{\Lambda}_{11} & \tilde{\Lambda}_{12} \\
0 & \Phi
\end{array}\right]\left[\begin{array}{c}
\bar{h}_{1}(k) \\
\sigma_{h}(k)
\end{array}\right]+\left[\begin{array}{c}
\tilde{v}_{1}(k) \\
0
\end{array}\right] .
$$

Obviously, the eigenvalues of $\Phi$ are designed to be stable. In addition, it can be easily proved that $\tilde{\Lambda}_{11}=\bar{\Lambda}_{11}-\bar{\Lambda}_{12} \bar{S}_{2}^{-1} \bar{S}_{1}$ which is designed to be a stable matrix by (17). Consequently, the system (20) is stable under the control law (22).

Remark 2: As seen from (21) and (23), the states $\sigma_{h}(k)$ in sliding surface are not influenced by the boundary conditions. However, broadly speaking, the boundary conditions have an obvious effect on the dynamics of the total states and, in addition, the final value that the states approach to. The bound of states will be found in Section V when the system (7) is assumed to have boundary conditions and an additional uncertainty term.

It should be mentioned that instead of control law (22), another direct method is also possible to obtain the sliding control law [18], [20]. Assuming that matrices $\bar{S}_{1}$ and $\bar{S}_{2}$ have been designed (by for instance LQR design) such that the reduced order dynamics (17) is stable. Now, from (21), (15) and (7) we have

$$
\Phi \sigma_{h}(k)=S[\Lambda H(k)+B U(k)+v(k)],
$$

where $S$ is defined in (18). Thus, the control law can be determined by

$$
U(k)=(S B)^{-1}[\Phi S H(k)-S \Lambda H(k)-S v(k)] .
$$

This control law is called direct control law which can be obtained directly after computing sliding matrix $S$.

\section{STRONG STABILITY FOR DISCRETE-TIME SYSTEMS}

The stability studied in Theorem 1 is based on the notion of asymptotic stability. To analysis the effect of uncertainty, in this section, another notion of stability referred to as strong asymptotic stability or briefly strong stability is studied.

Definition 1: Consider the following LTI discrete-time system

$$
x(k+1)=A x(k), \quad k \geq 0, \quad x \in R^{n} .
$$

The system (26) is strong asymptotically stable if and only if $\|x(k+1)\|<\|x(k)\|$, for all $k>0: x(k) \neq 0$.

Lemma 1 ( [23]): The system in (26) is strong asymptotically stable if and only if

$$
\|A\|<1 \text {. }
$$

Although it is clear that all the systems are not strong stable with respect to Euclidean norm, in the following it will be shown that each asymptotically stable system is strong asymptotically stable with respect to a specific norm.
Lemma 2 ( [23]): For each asymptotically stable LTI discrete-time system (26) there exists a similarity transformation matrix $T$, such that $\left\|T A T^{-1}\right\|<1$.

Lemma 3 ( [22]): If $T$ is a nonsingular similarity transformation matrix then

$$
\|A\|_{T} \triangleq\left\|T A T^{-1}\right\|,
$$

is a matrix norm.

Thus, it should be noted that $\left\|T A T^{-1}\right\|$ can be assumed as a special norm $(T-$ norm $)$ of the matrix $A$. Lemmas 2 and 3 ensure that for every asymptotically stable system there exists a specific norm such that, under that norm, the closed-loop system is strong asymptotically stable [23]. The issue of strong asymptotic stability for uncertain systems will be studied in the next section. More importantly, a specific similarity transformation $T$ such that $\left\|T A T^{-1}\right\|<1$ which is known as the balancing transformation is introduced in [24].

Lemma 4: Define a vector norm $\|x\|_{T}$ as

$$
\|x\|_{T} \triangleq\|T x\|
$$

Then, the matrix norm in (28) is an induced norm for the vector norm in (29).

Proof: It can be shown that for every $A \in R^{n \times n}$,

$$
\begin{aligned}
& \max _{\|x\|_{T}=1}\|A x\|_{T}=\max _{\|x\|_{T}=1}\|T A x\|= \\
& \max _{\|T x\|=1}\left\|T A T^{-1} T x\right\|=\left\|T A T^{-1}\right\|=\|A\|_{T} .
\end{aligned}
$$

Remark 3: Moreover, for every vector $x \in R^{n}$ we have

$$
\|T\|^{-1}\|x\|_{T} \leq\|x\| \leq\left\|T^{-1}\right\|\|x\|_{T} .
$$

Besides, for every matrix $A \in R^{n \times n}$,

$$
\operatorname{cond}(T)^{-1}\|A\|_{T} \leq\|A\| \leq \operatorname{cond}(T)\|A\|_{T},
$$

where $\operatorname{cond}(T)=\|T\|\left\|T^{-1}\right\|$ is the condition number of the transformation matrix $T$.

\section{DSMC FOR UNCERTAIN FORM}

Generally, the discrepancy between the original $P D E$ model (1) and its discrete 1D vectorial approximation (7) depends on the step sizes $\Delta x$ and $\Delta t$. This matter can be assumed as uncertainty in the state space (7). It means that we have

$$
H(k+1)=\Lambda H(k)+B U(k)+v(k)+f(k),
$$

where the uncertain term $f(k) \in R^{(n-1)}$. We consider the following general uncertainty

$$
f(k)=\Delta H(k)+d_{k},
$$

where $\Delta$ shows the unknown uncertainty with the bound $\|\Delta\|<\alpha_{0}(\|$.$\| is the induced Euclidean or induced spectral$ norm). Moreover, the term $d_{k} \in R^{(n-1)}$, indicates the external disturbance and it is assumed that $\left\|d_{k}\right\|<\beta_{0}$, where $\beta_{0}$ is a known positive constant. As a result, we can write

$$
\|f(k)\|<\alpha_{0}\|H(k)\|+\beta_{0} .
$$


Thus, with the control law (22) the closed-loop system is

$$
\begin{array}{r}
{\left[\begin{array}{c}
\bar{h}_{1}(k+1) \\
\sigma_{h}(k+1)
\end{array}\right]=\left[\begin{array}{cc}
\tilde{\Lambda}_{11} & \tilde{\Lambda}_{12} \\
0 & \Phi
\end{array}\right]\left[\begin{array}{l}
\bar{h}_{1}(k) \\
\sigma_{h}(k)
\end{array}\right]+} \\
{\left[\begin{array}{c}
\tilde{v}_{1}(k) \\
0
\end{array}\right]+\left[\begin{array}{l}
\tilde{f}_{1}(k) \\
\tilde{f}_{2}(k)
\end{array}\right],}
\end{array}
$$

where $\tilde{f}(k)=T_{s} T_{r} f(k)$ and also it is obvious that $\tilde{f}_{2}(k)=$ $S f(k)$; here $S$ is defined in (18). Now, back to the original coordinate $H(k)$ from (36), it is obtained that

$$
H(k+1)=\Lambda_{e q} H(k)+v_{e q}(k)+f(k),
$$

where

$\Lambda_{e q}=T_{r}^{-1} T_{s}^{-1}\left[\begin{array}{cc}\tilde{\Lambda}_{11} & \tilde{\Lambda}_{12} \\ 0 & \Phi\end{array}\right] T_{s} T_{r}, v_{e q}(k)=T_{r}^{-1} T_{s}^{-1}\left[\begin{array}{c}\tilde{v}_{1}(k) \\ 0\end{array}\right]$

Here, a new theoretical justification of the robustness of the closed-loop system against uncertainty based on the strong stability notion represented in Section IV, is given. According to Lemmas 2 and 3, it is known that a special induced norm $\left(T\right.$-norm) of the closed loop matrix $\left(\Lambda_{e q}\right)$ is necessarily smaller than 1. Hence, from (37) and (38), one can write that

$$
\|H(k+1)\|_{T} \leq\left\|\Lambda_{e q}\right\|_{T}\|H(k)\|_{T}+\left\|v_{e q}(k)\right\|_{T}+\|f(k)\|_{T} .
$$

Here, the induced norm $\left\|\Lambda_{e q}\right\|_{T}$ is used as proved in Lemma 4. Besides, the bound of uncertainty in this new coordinate can be shown as

$$
\begin{array}{r}
\|f(k)\|_{T} \leq\left\|T \Delta T^{-1} T H(k)\right\|+\left\|T d_{k}\right\| \leq \\
\|T\|\|\Delta\|\left\|T^{-1}\right\|\|T H(k)\|+\|T\|\left\|d_{k}\right\| \leq \\
\alpha_{0}\|T\|\left\|T^{-1}\right\|\|H(k)\|_{T}+\beta_{0}\|T\| .
\end{array}
$$

Introducing $\alpha=\operatorname{cond}(T) \alpha_{0}$ and $\beta=\|T\| \beta_{0}$, the following theorem is represented to consider the bound on system states under $T$-norm and in the presence of uncertainty of the form (34).

Theorem 2: Consider the uncertain system (37) with uncertainty defined in (34). If we have $\Psi+\alpha<1$, where $\Psi=\left\|\Lambda_{e q}\right\|_{T}$, then for any $\varepsilon>0$, the states of the closedloop system are bounded under $T$-norm by $\rho_{\varepsilon}=\frac{\beta_{1}}{1-\Psi-\alpha}+\varepsilon$, when $k \rightarrow \infty$, where $\beta_{1}=\beta+\max _{k}\left(\left\|v_{e q}(k)\right\|_{T}\right)$. Moreover, the distance from sliding surface is bounded by $S_{T} \rho_{\varepsilon}$, where $S_{T}=\|S\|_{T}$.

Proof: From (39) and (40), we derive

$$
\|H(k+1)\|_{T}<(\Psi+\alpha)\|H(k)\|_{T}+\beta_{1} .
$$

Thus, it can be shown that

$$
\|H(k)\|_{T}<(\Psi+\alpha)^{k}\|H(0)\|_{T}+\sum_{i=0}^{k-1}(\Psi+\alpha)^{i} \beta_{1}
$$

As a result, when $\Psi+\alpha<1$,

$$
\|H(k)\|_{T}<(\Psi+\alpha)^{k}\|H(0)\|_{T}+\frac{\beta_{1}}{1-\Psi-\alpha} .
$$

Hence, one can derive that

$$
\begin{aligned}
& \forall \varepsilon>0, \quad \exists K>0, \text { s.t. } \\
& \qquad\|H(k)\|_{T}<\rho_{\varepsilon}=\varepsilon+\frac{\beta_{1}}{1-\Psi-\alpha}, \forall k>K .
\end{aligned}
$$

Besides, from (15) it can be concluded that

$$
\left\|\sigma_{h}(k)\right\|_{T} \leq S_{T}\|H(k)\|_{T}
$$

Consequently, the bound on the distance from sliding surface is $S_{T} \rho_{\varepsilon}$.

Remark 4 (disturbance only): When there is no modelbased uncertainty and the system includes only disturbance $d_{k}$, the parameter $\alpha$ is zero in (35). As a consequence, the states of the closed loop system will be bounded by $\rho_{\varepsilon}=\frac{\beta_{1}}{1-\Psi}+\varepsilon$.

Remark 5: To find the bound in (44) in terms of Euclidean norm $\left(\hat{\rho}_{\varepsilon}\right)$, one can simply obtain from (44) and (31) that

$$
\|H(k)\|<\hat{\rho}_{\varepsilon}=\varepsilon+\frac{\operatorname{cond}(T)\left[\beta_{0}+\max _{k}\left(\left\|v_{e q}(k)\right\|\right)\right]}{1-\Psi-\alpha} .
$$

\section{SIMULATION RESULTS}

We consider the heat flow equation (1) over $0 \leq t \leq$ $40(\mathrm{sec})$ and $0 \leq x \leq 42(\mathrm{~m})$ with $\Upsilon=1\left(\frac{\mathrm{m}^{2}}{\mathrm{sec}}\right)$. Using the discretization equations (5) and (6), with $\Delta t=1(\mathrm{sec})$ and $\Delta x=2(m)$, we have $\kappa=0.25$. Since $0 \leq \kappa \leq 0.5$ the obtained discrete scheme will be stable. In addition, it is supposed that the state initial conditions are equal to 1 , and also initial control inputs are equal to 0 . Moreover, it is supposed that the state boundary conditions (2) and boundary control inputs are set to zero. Also, it is assumed that the control input is in spatially piece-wise constant form with the control input matrix of the form $B_{1}$ in (9) with $\mu=1$. As a result, control is defined only at the space points $1,3,5, \cdots, 19$ on the grid. It is clear that the pair $(\Lambda, B)$ is controllable. To compute orthogonal matrix $T_{r}$ we have used Matlab $Q R$ command. The matrix $\bar{S}_{2} \in R^{10 \times 10}$ is chosen as $\bar{B}_{2}^{-1}$ and in addition matrix $\bar{S}_{1} \in R^{10 \times 10}$ is determined by LQR design with state weighting of $10 I_{10}$ and control weighting of $10^{2} I_{10}$. Eventually, matrix $S$ is achieved from equation (18). Furthermore, it is assumed that $f(k)=0.2 H(k)+0.2 \sin \left(\frac{H(k)}{3 \pi}\right) \cos \left(\frac{H(k)}{2 \pi}\right)$. Here, $\Phi=0.3 I_{10}$ is used in control law (22) and the results of applying SMC for discretized state space (7) are given in Figures 1-3. These figures show the system states, 2D control effort and also 2-norm of control law and states, respectively. Since the 2D figures are not good indexes for studying the performance of controller, 2-norm of vectors $U(k)$ and $H(k)$ are given in Figure 3 as an index to study the performance of DSMC.

\section{CONCLUSION}

In this paper, we have developed new results on the application of DSMC to a kind of dynamics described by PDEs. Firstly, the underlying PDE is discretized by the use of Taylor's expansion and then the attained 2D discrete system is converted to 1D vector form. As a result of the proposed method of this paper, space variable is hidden in the final 1D process structure. Secondly, to stabilize an unstable discretized 1D vectorial form, DSMC is applied to our problem successfully. Next, the discretizing error which is modelled in uncertainty term is studied. To this end, using notion of 


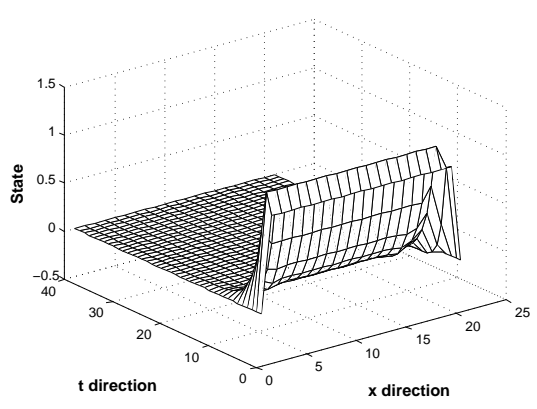

Fig. 1. States for spatially piece-wise constant controller, with $\Phi=0.3 I_{10}$

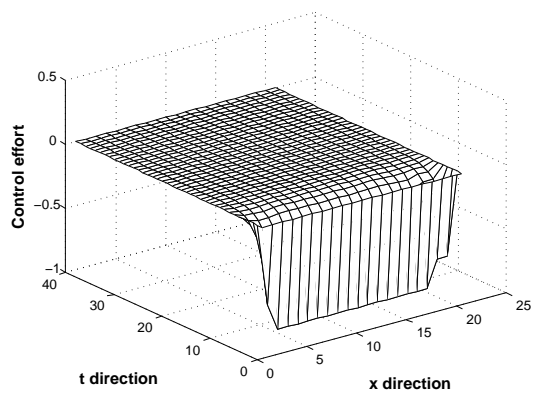

Fig. 2. Spatially piece-wise constant control effort, with $\Phi=0.3 I_{10}$

strong stability and similarity transformation matrices a new framework to analyze the state bound in the presence of general uncertainty is proposed. Furthermore, since there are space constraints on the location of actuators (spatially piecewise constant, sparse form or boundary control), a general strategy which can handle all these schemes is proposed in this paper. The simulation results confirm that the proposed DSMC is efficient and effective for the underlying PDEs.

\section{REFERENCES}

[1] Y. Ou et al.,Towards model-based current profile control at DIII-D, Fusion Engineering and Design, vol. 82, 2007, pp 1153-1160.

[2] M. Krstic, "Compensating a String PDE in the Actuation or Sensing Path of an Unstable ODE", American Control Conference, USA, 2009, pp 4097-4102.
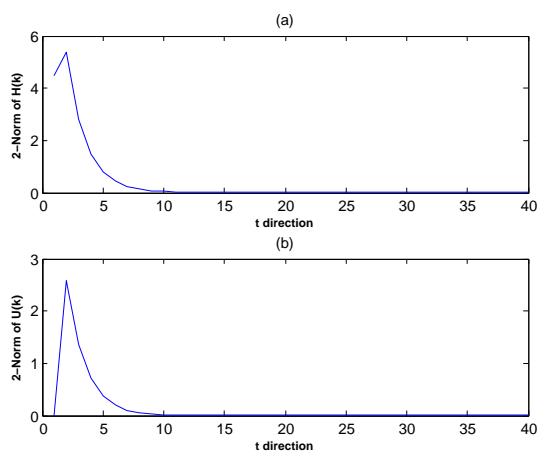

Fig. 3. 2-norm of the (a) state vector and (b) control effort with $\Phi=0.3 I_{10}$
[3] N. Bekiaris-Liberis, M. Krstic, Compensating the Distributed Effect of a Wave PDE in the Actuation or Sensing Path of MIMO LTI Systems, Systems and Control Letters, vol. 59, 2010, pp 713-719.

[4] B.S.J. Choi and K. Lee, Constrained digital regulation of hyperbolic PDE systems: A learning control approach, Korean Journal of Chemical Engineering, vol. 18, 2001, pp 606-611.

[5] C. Xu, R. Arastoo and E. Schuster, "On Iterative Learning Control of Parabolic Distributed Parameter Systems", in 17th Mediterranean Conference on Control and Automation, Greece, 2009, pp 510-515.

[6] K.L. Moore and Y.Q. Chen, "Iterative learning approach to a diffusion control problem in an irrigation application", in Proceedings of the 2006 IEEE International Conference on Mechatronics and Automation, China, 2006, pp 1329-1334.

[7] B. Cichy, K. Galkowski and E. Rogers, Iterative learning control for spatio-temporal dynamics using Crank-Nicholson discretization, Multidimensional Systems and Signal Processing, vol. 23, Issue 1-2, 2012, pp 185-208.

[8] V.I. Utkin, Variable structure systems with sliding modes, IEEE Trans. Automat. Contr., vol. AC-22, 1977, pp 212-222.

[9] M. Cheng, V. Radisavljevic, and W. Su, "Sliding Mode Boundary Control of Unstable Parabolic PDE Systems with Parameter Variations and Matched Disturbances", American Control Conference, USA, 2009, pp 4085-4090.

[10] S. Drakunov, E. Barbieri and D.A. Silver, Sliding Mode Control of a Heat Equation with Application to Arc Welding, Proceedings of the 1996 IEEE International Conference on Control Applications, Dearborn, 1996, pp 668-672.

[11] R. Courant, K. Friedrichs, and H. Lewy, On Partial Difference Equations of Mathematical Physics, IBM Journal 11, 1967, pp 215234. (English translation of the original work, Uber die Partiellen Differenzengleichungen der Mathe- matischen Physik, Math. Ann. 100, 1928, pp 32-74).

[12] C. Milosavljevic, General conditions for the existence of a quasisliding mode on the switching hyperplane in discrete variable structure systems, Automation and Remote Control, vol. 3, 1985, pp 36-44.

[13] P.J. Olver, Introduction to Partial Differential Equations, University of Minnesota, 2010.

[14] A. El Jai, Distributed systems analysis via sensors and actuators, Sensors and Actuators vol. 29, 1991, pp 1-11.

[15] H.N. Iordanou and B.W. Surgenor, Experimental evaluation of the robustness of discrete sliding mode control versus linear quadratic control, IEEE Transactions on Control Systems Technology, vol. 5(2), 1997, pp 254-260.

[16] A. Bartoszewicz, Discrete-time quasi-sliding-mode control strategies, IEEE Transactions on Industrial Electronics, vol. 45, 1998, pp 633637.

[17] S. Hui and S. H. Zak, On discrete-time variable structure sliding mode control, Systems and Control Letters, vol. 38, 1999, pp 283-288.

[18] Monsees G. Discrete-time sliding mode control, Ph.D. Thesis, Delft University of Technology, The Netherlands, 2002.

[19] V. Utkin, Sliding Modes in Control Optimization, Communications and Control Engineering Series, Springer-Verlag, London, 1992.

[20] S. K. Spurgeon, Hyperplane design techniques for discrete-time variable structure control systems, International Journal on Control vol. 55(2), 1992, pp 445-456.

[21] S.Z. Sarpturk, Y. Istefanopulos and O. Kaynak, On the stability of discrete-time sliding mode control systems, IEEE Transactions on Automatic Control vol. 32(10), 1987, pp 930-932.

[22] R.A. Horn and C.R. Johnson, Matrix Analysis, Cambridge University Press, 1985.

[23] G. Halikias, L. Dritsasb, A. Pantelousc and V. Tsoulkas, Strong stability of discrete-time systems, Linear Algebra and its Applications, vol. 436, 2012, pp 1890-1908.

[24] L. Pernebo, L.M. Silverman, Model reduction via balanced state-space representations, IEEE Trans. Automat. Control vol. 27 (2), 1982, pp 382-387.

[25] W. Su, S.V. Drakunov and U. Ozguner, An $\mathrm{O}\left(T^{2}\right)$ boundary layer in sliding mode for sampled-data systems, IEEE Transactions on Automatic Control vol. 45(3), 2000, pp 482-485.

[26] C. Edwards, S.K. Spurgeon, Sliding Mode Control: Theory and Applications, Taylor and Francis, London, 1998. 